\title{
Libanais et Syriens au Brésil (1880-1950)
}

\section{Oswaldo Truzzi}

\section{OpenEdition}

Journals

Édition électronique

URL : https://journals.openedition.org/remi/1694

DOI : $10.4000 /$ remi. 1694

ISSN : 1777-5418

\section{Éditeur}

Université de Poitiers

\section{Édition imprimée}

Date de publication : 1 mars 2002

Pagination : 123-147

ISBN : 2-911627-30-X

ISSN : 0765-0752

\section{Référence électronique}

Oswaldo Truzzi, «Libanais et Syriens au Brésil (1880-1950) », Revue européenne des migrations internationales [En ligne], vol. 18 - $\mathrm{n}^{\circ} 1$ | 2002, mis en ligne le 09 juin 2006, consulté le 15 avril 2022. URL : http://journals.openedition.org/remi/1694 ; DOI : https://doi.org/10.4000/remi.1694

Ce document a été généré automatiquement le 15 avril 2022

(C) Université de Poitiers 


\title{
Libanais et Syriens au Brésil (1880-1950)
}

\author{
Oswaldo Truzzi
}

1 Le départ de milliers d'immigrants d'origine libanaise et syrienne vers le Brésil a commencé alors que la Syrie et le Liban se trouvaient encore sous domination ottomane $^{1}$ (Truzzi, 1997a). C'est à partir des deux dernières décennies du xix siècle que commencent à arriver au Brésil des vagues significatives d'immigrants en provenance de cette région que l'on appelait alors la grande Syrie².

2 Cet article a pour but de repérer les trajectoires de ces immigrants au Brésil au cours de la période 1880-1950. Après avoir souligné les principales raisons de l'émigration, je discuterai brièvement du volume des contingents et de leur distribution géographique au Brésil. Ensuite, je décrirai les conditions de l'insertion tout d'abord en tant que colporteurs, puis par la mise en place d'un réseau commercial ethnique. Je parlerai alors de l'image et de l'identité du groupe par rapport au reste de la société, ainsi que des éléments de différenciation internes les plus importants. Enfin, je présenterai les lignes majeures de l'intégration des catégories plus favorisées nées au Brésil, qui exercent des professions libérales et ont bâti des carrières politiques.

L'arrivée des libanais et des syriens au brésil

3 L'émigration vers le Brésil s'inscrit dans le cadre général des mouvements de populations orientales, comme celles venant d'Europe, vers le continent américain. Les départs sont liés, d'une façon générale, à des facteurs de nature économique et démographique désagrégeant l'économie de subsistance établie autour des villages, qui vivaient quasiment en autosuffisance. Le progrès des transports maritimes et terrestres facilitant l'importation de biens manufacturés a miné la production locale des artisans indépendants; le développement des villes a également développé une production agricole destinée au commerce, portant atteinte à celle destinée à la subsistance. L'industrie textile, en grande partie à caractère familial, n'a pas résisté à la compétitivité des produits importés (Tannous, 1942).

4 La croissance démographique de la population, le caractère semi-désertique du climat et la petite taille des exploitations agricoles limitaient l'accès des jeunes à la propriété 
et les encourageait à émigrer. En outre, et à un degré moindre, des facteurs de nature politique et religieuse, liés à la désagrégation de l'Empire 0ttoman ou à des conflits entre fractions religieuses influencèrent ces départs ${ }^{3}$.

5 Les données sur les flux migratoires soient assez discutables, une partie appréciable des départs, notamment à partir de l'actuel Liban, auraient eu lieu clandestinement. Mais elles permettent de définir une première période d'émigration importante entre 1880 et $1930^{4}$. Les États-Unis surtout, mais aussi le Brésil et l'Argentine furent les destinations privilégiées de ces immigrants d'origine libanaise et syrienne. Au Brésil, l'immigration de Libanais et de Syriens est devenue importante au début du $\mathrm{xx}^{\mathrm{e}}$ siècle et a atteint son apogée à la veille de la Première Guerre mondiale (le nombre maximum d'entrées, 11 101, a été enregistré en 1913) pour ensuite s'interrompre durant le conflit, puis se stabiliser pendant les années vingt autour de cinq mille entrées annuelles. L'immigration a diminué après 1930, subissant la dépression économique et limitée par le système de quotas inspiré des États-Unis et adopté par le gouvernement brésilien.

6 Au Brésil, pendant longtemps les immigrants de la région ont été comptabilisés dans la catégorie "autres nationalités ", et c'est seulement à partir de 1908 et dans l'Etat de São Paulo que les services d'immigration enregistrèrent les immigrants selon leur nationalité (Turcs, Turcs-asiatiques, Libanais ou Syriens...). Entre 1908 et 1941 leur contingent a atteint $4 \%$ des 48326 immigrants entrés dans l'Etat, derrière les Portugais, les Espagnols, les Italiens, les Japonais et les Allemands.

7 Le fait est, qu'à partir des dernières décennies du XIX ${ }^{e}$ siècle, la réussite de quelques pionniers stimula l'émigration de façon notoire. La possibilité de gagner de l'argent bouleversa l'équilibre des villages. Les familles commencèrent à planifier le départ temporaire de leurs enfants en Amérique pour résoudre leurs problèmes financiers: celles qui ne le faisaient pas perdaient leur statut et leur prestige.

Deux faits illustrent de façon exemplaire leurs motivations. Le premier réside dans l'importance des transferts d'argent que les immigrants envoyaient au village afin d'acquérir des terres, agrandir les propriétés rurales de leur famille pour qu'elle puisse en vivre. La poste devint une institution prestigieuse puisqu'elle apportait des nouvelles et de l'argent à ceux qui étaient restés sur place. Le second se réfère au caractère temporaire de l'immigration telle qu'elle fut imaginée à son début: les immigrants calculaient que quelques années passées en Amérique seraient suffisantes pour assurer la prospérité de leurs familles. D'où une forte majorité d'hommes célibataires parmi les émigrants.

9 Au Brésil, aucune autre œuvre littéraire n'a montré, avec autant de fidélité, les liens entre les immigrants et ceux restés au pays, que le roman gai et émouvant de Emil Farhat, «Dinheiro na Estrada: uma saga de imigrantes », écrit à partir de lettres échangées entre l'auteur, lui-même immigrant, et sa mère, institutrice à Kafaarchima, village proche de Beyrouth, inconsolable du départ de ses six enfants pour le Brésil.

«Et maintenant, étant dans cette mine Brésil depuis vingt ans, Iskândar n'a pas encore rempli son sac avec cette fortune dont il parlait tellement ? Et alors, pourquoi il ne revient pas ? Et toi ? Il est l'aîné, mais je t'ai autorisé à lui tordre les oreilles. Oui, de cette façon. Alors, pourquoi tu ne l'as pas fait. Vous êtes tous une bande de vauriens. Vous vous êtes mis d'accord pour me laisser de côté, pleurant dans un coin de la maison. Pleurant cachée pour que vos frères et sœurs ne le voient pas. Ils meurent de jalousie des larmes que je verse à cause de vous. 
Pourquoi cette tête d'aubergine fanée ? Vos frères et sœurs, ceux d'ici, ont raison. Alors, cela n'est pas une chose qui se fait? Vous vous êtes attachés à ce Brésil, à cette Amérique, et terminé. Mon Dieu ! Je Lui demande qu'Il ne me laisse pas sentir le temps passer. Pour moi, vous êtes partis hier. Avant-hier. Parfois, je réussis à voir clairement la tête de tous. Un par un. Iskândar, avec sa grande moustache, seulement pour cacher sa tête de gamin effrayé. In-Hula, électrique, pressé, toujours se brûlant avec la soupe chaude. Muzáref, avec ses doigts teintés qu'il remue sans arrêt. Il dit que c'est un exercice pour compter l'argent, pour se frotter les mains au lieu de dire adieu, au moment du départ. Ziad récitant des vers pour un adversaire qui l'a défié et qui perdait toujours. Pour des spectateurs qui l'applaudissent. Nazira la pleureuse, voulant y aller et ne voulant plus, désireuse d'y aller, moi seule, connaissant le motif. Pour se marier. Elle s'est mariée ? Mais, pourquoi on ne m'a rien dit avant? Je deviens sourde, mais pas pour écouter les nouvelles de mes enfants. Des nouvelles de son enfant, on les écoute toujours avant même que les bouches ne s'ouvrent.

Et toi, l'orgueilleux, le vaniteux, le batailleur Tauil? «Je vais au Brésil et je les ramènerai tous par la peau du cou. » Qui tu as ramené ? Personne! » (Farhat, 1987 : 72)

10 Un leader de la mission presbytérienne de la région se plaignit que «la fièvre migratoire ne présente aucun indice de diminution. C'est même devenu une manie. Elle a enlevé de nos églises quelques-uns de nos membres les plus utiles; plusieurs de nos professeurs sont inquiets. Un analphabète s'en va en Amérique et dans les six mois qui suivent il envoie un chèque de 300 ou 400 dollars, plus que ce que gagne un professeur ou un pasteur en deux ans. » (Knowlton, $1961: 29$ )

11 L'intensité du désir de partir ne doit pas, cependant, déformer l'essentiel du processus migratoire. La majorité de ceux qui ont émigré ne l'a pas fait à la suite d'une décision individuelle, mais soutenu par la famille ou par un ensemble de compatriotes. Dans le cadre familial, la condition pour que certains émigrent était que les autres parents assument le travail de l'exploitation. De plus, le transfert massif d'argent ajouté au caractère initialement temporaire de cette émigration est loin de donner l'image d'un migrant aventurier irresponsable ne recherchant qu'un gain personnel. L'interprétation la plus fiable serait de considérer cette migration comme un mouvement d'individus tenus par des liens familiaux et travaillant pour assurer les priorités du groupe familial au pays. Que ce soit à travers leurs envois d'argent ou par l'établissement d'une vie familiale dans le nouveau monde, les migrants cherchaient à sortir leurs familles de situations économiques difficiles.

Les débuts

12 Malheureusement, il n'existe pas de statistiques sur la répartition des Syro-Libanais au Brésil au début du siècle. Ce que l'on sait, c'est que dans les premières années existait trois principaux centres d'attraction au Brésil : l'Amazonie, São Paulo et Rio de Janeiro.

13 Alors qu'arrivaient les premiers immigrants, l'Amazonie connaissait un boom du caoutchouc. Les colporteurs syriens ou libanais n'étaient pas seulement intéressés, comme les autres, par le caoutchouc, mais surtout par la vente de marchandises. En peu de temps, ils concentrèrent entre leurs mains le commerce du bassin amazonien. À partir des principaux centres où s'était implantée la colonie, comme celui près de l'église de Notre Dame des Remèdes de Manaus, ou encore à Belém, le colporteur se déplaçait en bateau, négociant avec les habitants. marchandeurs) qui remontaient les rivières, remorqués par des gaiolas, sorte de grands 
bateaux, et s'engageaient dans un commerce dangereux, considéré comme illégal par les propriétaires des plantations de caoutchouc, désirant contrôler la production sur leur propriété. Le regatão, se dissimulant, marchandait directement avec les employés et faisait des affaires sous forme de troc. Type social et commercial surgissant des contingences de l'environnement, le regatão fut un défi permanent pour les navigateurs traditionnels et les propriétaires de caoutchouc qui avaient le monopole des rivières. Le regatão accomplissait de longs voyages, vendant à l'ouvrier des plantations de caoutchouc ses marchandises au comptant, et recevant en contrepartie du caoutchouc, l'échange se faisant toujours dans des endroits définis au préalable, connus des deux seules parties et jamais au port officiel de la propriété, où il aurait été facilement repéré.

15 De nombreux regatões étaient d'origine libanaise ou syrienne et exerçaient sur les populations, dispersées le long des principales rivières d'Amazonie, une véritable fascination, car ils apportaient des produits nouveaux et, en même temps, des nouvelles de la capitale. Ils parcouraient les rivières dans des bateaux de bois, couverts de paille ou de toile fermée sur les côtés par des morceaux de bois peint, et chargés de marchandises variées,

" depuis l'aiguille à coudre, l'hameçon, les bobines de fils de toutes les couleurs, les boutons, les bougies, l'eau de vie, les brodequins, les ligne de pêche, les éperviers, les boucles d'oreille, les bagues, les chaînes, les bracelets en métal, les chapeaux de paille, les pâtes, le cuir, les pantalons, les chemises, les caleçons, les balles de rifles, les fusils, les couteaux, les houes, les allumettes, les cigarettes de plusieurs marques, le savon en morceaux, les savonnettes parfumées, les moustiquaires, les hamacs du Ceará, les réchauds, les éventails, les purgatifs à base de plantes, le sel de mer, le propocloreto de mercure, les guitares, les flûtes et un monde de marchandises, toutes de très mauvaise qualité. » (Andrade, 1985a : 49)

16 Avec le temps, plusieurs des colporteurs réussirent à s'établir dans différentes villes de l'Amazonie ou même, à la fin du cycle du caoutchouc, à créer leurs propres magasins de caoutchouc, en particulier dans l'État de l'Acre et sur les rivières des régions isolées (Andrade, 1985b; Benchimol, 1985). Ainsi, de l'Acre au Pará, il n'y avait aucune ville où le commerce caractéristique des Libanais et des Syriens ne soit présent. Après la Première Guerre mondiale, la fin du cycle du caoutchouc détermina le départ de beaucoup de Syro-libanais pour São Paulo ou pour Rio, mais certains restèrent et construisirent un réseau commercial important pour la région, toujours actif de nos jours.

17 Les mines et les zones agricoles florissantes de l'État du Minas Gerais attirèrent aussi les immigrants. Ils s'établirent partout dans le Minas, jouant un rôle important dans le développement du commerce et de l'industrie. Dans plusieurs villages et villes, la quasitotalité du commerce de détail était entre leurs mains. Certains, enrichis, achetèrent des terres et devinrent fermiers et éleveurs de bétail, d'autres investirent leur argent dans des machines pour traiter le coton, dans des épiceries ou dans des entreprises industrielles agricoles.

Un des parcours les plus intéressants est celui d'Elias Aun, né dans la petite ville côtière de Damour, proche de Beyrouth, en 1904. Ses parents élevaient des vers à soie et exportaient leur production. Avec l'accroissement de la concurrence, la famille décida d'émigrer aux États-Unis, mais à Marseille, le père n'obtint pas son visa pour avoir subi une opération chirurgicale à l'œil. Aun avait 9 ans quand il arriva au Brésil. Il vécut 
dans des bidonvilles à São Paulo, jusqu'au jour où un compatriote invita la famille à installer un bar à Ibirá, dans l'intérieur de l'État de São Paulo, pour y vendre de l'eau de vie. Peu à peu, il apprit la comptabilité et offrit alors ses services à ses compatriotes possédant épiceries et petits magasins. En même temps, il commença à s'intéresser à la photographie. En 1927 il épousa la fille d'un Italien.

Avec la crise de 1929, plusieurs commerçants firent faillite et Aun se trouva sans travail. Il décida, alors de déménager à Belo-Horizonte, capitale de la province du Minas Gerais, où la colonie libanaise et syrienne avait concentré ses affaires aux environs de la rue Caetés. Il travailla comme vendeur, puis il trouva un emploi dans une papeterie, où il devint responsable du laboratoire photographique. En 1936, il installa son magasin de photos au centre ville. Ses affaires prospérèrent et, soixante ans plus tard, en 1992, Kodak lui rendit hommage comme étant le plus ancien représentant travaillant encore ; Aun avait 92 ans.

Une caractéristique fondamentale de la colonie libanaise au Brésil est sa répartition sur tout le territoire national. Leur travail de colporteurs amenaient les immigrants à construire leurs réseaux jusqu'aux contrées les plus lointaines, peu accessibles aux moyens de transports conventionnels. Les premiers Libanais, originaires des villes de Chiká, Djoune, Sarba, Barsa, Zouk Mickael, Daroun, Anfi et des autres villages des environs, arrivèrent au sud du pays en 1880. Certains débarquèrent à Montevideo et entrèrent au Brésil par voie terrestre. À Porto Alegre, les rues General Andrade Neves et Voluntários da Pátria étaient les préférées de la colonie, tant pour y commercer que pour $\mathrm{y}$ vivre. À Pelotas, durant le premier quart du $\mathrm{xx}^{\mathrm{e}}$ siècle, Felipe Mechereffe eut un rôle essentiel pour toute la collectivité syro-libanaise. Sur toute la frontière du sud, ravagée dans les années vingt par la Révolution Farroupilha, ce Libanais devint une référence pour tous les vieux colporteurs; ceux-ci se souviennent encore avec plaisir et vénération de cet homme toujours prêt à aider ses compatriotes qui affluaient de partout. Il a même abrité chez lui presque quarante colporteurs, leur prêtant des marchandises, plusieurs d'entre eux finissant par établir leur propre commerce en différentes villes de l'intérieur de l'État du Rio Grande do Sul.

Un autre point important de concentration était la ville de Rio de Janeiro, qui, jusqu'à 1960, fut le siège de la capitale fédérale et où la colonie s'était concentrée aux alentours des rues da Alfândega, Senhor dos Passos et Buenos Aires. De nombreux immigrants logèrent à l'Hôtel Boueri, un bâtiment de deux étages, Place de la République. Ils reçurent de leurs compatriotes toutes les informations nécessaires pour la commande d'une armoire ambulante. En général, ces caisses, équipées de ceintures en cuir qui s'attachaient au dos du vendeur mesuraient 2 mètres de hauteur et 1,20 m de largeur, mais il y en avait de plusieurs dimensions, selon la taille du colporteur.

Au cours des années, des Juifs, des Chinois, des Coréens etc... - en plus des Portugais établis à l'origine - s'installèrent aussi dans le même quartier, devenue le centre d'un commerce vigoureux et varié; mélange de marchandises colorées, présentées avec différents accents. Plus récemment, au début des années soixante s'est créée l'association S.A.A.R.A. (Société des Amis des Alentours de la Rue de Alfândega), sigle devenu très populaire parmi les habitants de la ville. "Le mélange ethnique fait vivre ensemble au S.A.A.R.A. une grande variété de religions. Les Syriens et les Libanais sont, en majorité, des chrétiens maronites ou grecs-orthodoxes et, dans une moindre proportion, des musulmans. Les Juifs ont déjà eu une synagogue dans la région qui, aujourd'hui, n'existe plus. Les Chinois se partagent entre bouddhistes et chrétiens. » (Worcman, 1996) 

dominant depuis l'époque coloniale, mais il est intéressant de noter comment les Libanais maronites ont redéfini la tradition d'une de ces églises, celle de Saint Gonçalo Garcia, construite en 1758. À partir de 1850, la paroisse plaça sous sa protection la confrérie de Saint-Georges, rendant plus populaire le culte de ce saint que celui de Saint-Gonçalo. L'église est devenue fameuse sous le nom d'Église de Saint Georges. Or, Saint-Georges, "né à Cappadocia, est le saint d'un grand nombre d'immigrants libanais. Au S.A.A.R.A. il y avait beaucoup de magasins montrant avec ostentation son image décorée de fleurs et de rubans. Tous les ans, le 23 avril, la fête de Saint Georges est commémorée avec des coups de canons à l'aube et des messes durant toute la journée. » (Worcman, 1996)

Dans d'autres capitales régionales, il y eut des concentrations similaires, bien que dans des proportions inférieures. Au fil des années, les Libanais, les Syriens et leurs descendants s'insérèrent à tous les niveaux de la structure commerciale de plusieurs villes du pays, cherchant à s'installer dans les zones centrales de la ville, souvent proche de la gare ou des marchés, afin de profiter de la clientèle de passage.

作 1920 et de 1940 indiquent le nombre d'étrangers dans tout le pays, permettant, pour la première fois, de présenter la distribution régionale des Syriens et des Libanais au Brésil. Le premier recensement fit apparaître que presque $40 \%$ des quelques 20000 "Turcs asiatiques» du Brésil se trouvaient à São Paulo où ils représentaient la cinquième nationalité la plus nombreuse de l'État. Vingt ans plus tard, plus de la moitié du contingent national se trouvait dans cet État.

Tableau 1 : Libanais et Syriens dans les États du Brésil

\begin{tabular}{|l|r|r|r|c|}
\hline & \multicolumn{2}{|c|}{1920} & \multicolumn{2}{c|}{1940} \\
\hline État & Nombre & $\%$ & Nombre & $\%$ \\
\hline São Paulo & 19285 & 38,4 & 23948 & 49,3 \\
Rio de Janeiro (District Fédéral) & 9321 & 18,5 & 9051 & 18,6 \\
Minas Gerais & 8684 & 17,3 & 5902 & 12,1 \\
Autres États & 12956 & 25,8 & 9713 & 20,0 \\
\hline Brésil & 50246 & 100,0 & 48614 & 100,0 \\
\hline
\end{tabular}

Source : Recensements du Brésil, 1920 et 1940

En 1934, un recensement réalisé à São Paulo mit en évidence que plus d'un tiers de ceux qui vivaient dans l'État habitait la capitale. La rue 25 de Março, près du marché, qui depuis le début du siècle était la localisation pionnière du commerce de tissus et de la mercerie dans la capitale, attira l'attention d'un observateur comme un endroit « où la cacahuète grillée est remplacée par la graine de citrouille, où le quibe, sous toutes ses formes, surpasse le typique plat brésilien de haricots avec du riz ... L'ambiance est franchement syrienne. Il y a des libraires qui vendent seulement des livres écrits en arabe. On écoute constamment de la musique typique et des chansons plaintives et sentimentales chantées par les meilleures voix de l'Orient. Dans les pâtisseries et dans les cafés, les radios, en général, sont branchées sur les stations qui transmettent de la musique arabe et les clients parlent plus en langue étrangère que dans la langue du pays » (Araujo, $1940: 231$ ).

À mesure que le siècle avançait, l'image « d'hommes voués au commerce » se fixait. Ils étaient devenus experts dans certains secteurs qui, à l'époque, étaient importants, ayant compris que l'origine des affaires consistait à attirer la consommation populaire. Des auteurs comme Diegues Jr. ont affirmé que «quand un turc arrive dans une rue et 
s'installe pour développer une activité commerciale, tout de suite, elle se transforme; elle gagne de nouvelles couleurs, des couleurs ethniques (...) Les vitrines de quincailleries, les chemises pendues, les savonnettes attachées par des cordes, les cartables, les jouets d'enfants, enfin, la variété de coloris, d'objets exposés donnent tout de suite à la rue son caractère syrien » (Diegues Jr., $1966: 82$ ).

Cette histoire, riche en drames personnels, mais, en général bien réussie, débute avec la singulière image du colporteur ${ }^{5}$.

Curieusement, dans leur pays d'origine, cette vocation pour le commerce n'était pas si prononcée : la majorité des Syriens et des Libanais qui sont arrivés au Brésil au début de la vague migratoire était des agriculteurs de petites propriétés familiales, ou, dans certains cas, des artisans (Knowlton, 1961; Tannous, 1942). Il y a des informations raisonnablement fondées, assurant que le métier de colporteur était plus fréquemment exercé dans leur région d'origine par des Grecs, des Arméniens et des Juifs (Miller, 1981).

30 Pourquoi, alors, l'immigrant est-il devenu colporteur? L'élément fondamental pour comprendre l'insertion professionnelle dans sa nouvelle patrie réside dans le contraste entre les caractéristiques de la structure agraire dans le pays d'origine et celles du Brésil. Généralement issus de familles propriétaires de petits lopins de terre, ces immigrants se retrouvèrent, surtout à São Paulo, confrontés à un système de grandes propriétés. Face à une structure agraire concentrée, ils auraient dû travailler comme salariés pendant au moins une génération pour pouvoir acheter une terre qui les maintienne dans leurs activités d'origine. Dans les premières phases de l'immigration, certains sont allés travailler dans des fermes, mais sont partis au bout de quelques mois pour les villes proches, découragés par le traitement reçu et par l'absence de perspectives d'améliorer leur existence. Ces témoignages ont contribué à éloigner les autres immigrants de l'agriculture (Knowlton, 1961).

31 Durant la période 1908-1939, les données relatives à la profession des immigrants recensés à leur arrivée au Port de Santos indiquent que seulement $18 \%$ de Libanais et de Syriens se présentaient comme agriculteurs, probablement en raison de leur intention de travailler dans le commerce et de l'insertion préalable de membres de leurs familles ou de compatriotes.

Célibataires et souvent déterminés à rentrer dans leur pays d'origine après avoir épargné durant quelques années un capital destiné à leur donner de meilleures conditions de vie, les immigrants n'hésitèrent pas, dans leur grande majorité, à choisir une activité qui leur permettait de travailler pour eux-mêmes, échappant ainsi aux difficiles conditions de vie des salariés agricoles ou des ouvriers. Comme, en général, ils arrivaient sans aucun capital, l'activité la plus facilement envisagée était celle de colporteur, afin « de ne pas s'assujettir à des occupations serviles » (Kurban, 1933).

Cette vocation commerciale n'est pas le fait d'une simple insertion en milieu urbain : en premier lieu, la zone rurale a représenté la base des activités de colportage et, en deuxième lieu, ces immigrants n'ont pas, en dehors du commerce, exercé d'autres occupations typiquement urbaines (Ellis Jr., 1934). Deffontaines a observé que, depuis la fin du siècle dernier, «le domaine de travail des colporteurs s'est agrandi considérablement car ils représentaient, pour les ouvriers agricoles une heureuse concurrence au magasin $d u$ patron» (Deffontaines, $1936: 27$ ). 
Les colporteurs pénétrèrent dans les campagnes, parcoururent les fermes où ils étaient bien reçus par les agriculteurs qui préféraient commercer avec eux car les conditions de paiement étaient bien plus acceptables, et les achats faits en dehors du magasin du « patron » les rendaient moins dépendants. De ce fait, la population rurale constitua un important marché. La dispersion des Libanais et des Syriens sur tout le territoire national explique l'établissement de plusieurs familles dans de petits villages éparpillés de l'intérieur brésilien. Ils s'enracinèrent dans presque toutes les régions du pays.

L'activité de colporteur offrait plusieurs avantages, mais surtout elle n'exigeait pas d'habiletés spécifiques ou d'investissements. Ils commençaient en portant les panières et les énormes valises de ceux qui étaient déjà du métier et après avoir acquis quelques rudiments linguistiques, ils partaient seuls. Il y avait toujours la possibilité de remplir un plateau ou une valise de quincailleries variées et de les vendre dans les quartiers de la capitale, à l'intérieur du pays, ou dans les campagnes avides de nouveautés commerciales. Mais cette facilité d'installation ne doit pas masquer la dureté, la pénibilité du travail.

"Tôt le matin, les colporteurs sortaient, parcourant les rues, cherchant les maisons, supportant la chaleur, le froid et la pluie, apportant le pain et achetant n'importe quoi, de préférence $d u$ fromage et des bananes, pour l'unique repas du jour. Le soir, ils revenaient avec le gain du jour, très fatigués et faisaient les comptes avec le patron. Les bénéfices journaliers étaient chaque jour crédités au vendeur et c'est de cette manière que plusieurs ont formé leur capital initial pour devenir, à leur tour, commerçants et grossistes. Si les ventes au centre de la ville diminuaient, ils allaient en banlieue, s'éloignant graduellement, jusqu'à arriver dans les villages et les fermes des régions de l'intérieur du pays, toujours en vagues croissantes. Il y eut des colporteurs entreprenant des voyages en portant sur leur dos des caisses de quatre-vingts à cent kilos et qui épuisaient leur stock entre l'allée et le retour entre deux capitales. Au fur et à mesure que les affaires prospéraient, ils louaient des porteurs et, plus tard achetaient des ânes. " (Duoun, 1944 : 94).

De toute façon, cette activité était un bon début sur cette nouvelle terre. Le plus important était qu'après quelques années de dur labeur, il leur était possible d'accumuler un capital, ce qui n'était pas le cas des agriculteurs ou des ouvriers de l'époque. Le chômage, par exemple, une de leurs craintes en émigrant, ne les a jamais menacés. Les colporteurs, bien que plus « libres » et « éloignés » du tissu économique et social, n'ont jamais connu de limites à leur ascension sociale comme les ouvriers agricoles ou de l'industrie. Ils avaient devant eux un horizon ouvert .

Les colporteurs travaillaient, en général, pour des compatriotes bien établis qui leur prêtaient les marchandises. Le paiement pouvait donc avoir lieu après la vente d'une partie des produits. Le fournisseur était souvent un commerçant qui avait lui-même été colporteur et qui avait réussi à ouvrir son magasin. Il possédait une grande expérience $\mathrm{du}$ travail ce qui, d'une certaine façon, facilitait leurs rapports. Le colporteur pouvait aussi être quelqu'un de la famille, ce qui resserrait plus encore ces liens.

Travaillant avec ténacité et dépensant le moins possible pour survivre, les colporteurs étaient presque certain d'acquérir un capital ; et, au moins jusqu'à la fin de la Première Guerre mondiale, nombreux furent ceux qui ont dû faire un tel calcul, rêvant d'un retour réussi dans leur pays d'origine. Certains sont rentrés chez eux. Mais la majorité d'entre eux, arrivés célibataires, et rentrés après avoir gagné de l'argent, comparèrent les possibilités offertes par le Brésil et celles du pays natal, puis choisirent de constituer une famille et d'émigrer à nouveau. D'où l'habitude très commune entre pionniers de retourner dans la terre d'origine pour s'y marier. 
L'affirmation comme commerçants

39 À São Paulo en 1885, un groupe de colporteurs travaillait sur la place du marché. Célibataires et pauvres, ils habitaient dans des pensions ou des logements bon marché ou dans des sous-sols de vieux bâtiments (Araujo, 1940). Les immigrants «travaillaient d'abord comme assistant, transportant de lourdes caisses en bois pleines d'articles de mercerie sur leurs dos; ces caisses mesuraient quarante par cinquante par quatre-vingts centimètres; le propriétaire de l'affaire portait un panier plein d'articles de mercerie et, dans l'autre main, une crécelle qui annonçait sa présence, en produisant un son caractéristique et pénétrant " (Greiber et alii, 1999 : 81).

Le développement de la colonie libanaise et syrienne dans la rue 25 de Março résidait dans le fait que les pionniers s'y étaient installés d'abord, suivis par les familles et les amis qui arrivaient. En général les immigrants se dirigeaient vers cet endroit, ce qui leur permettait de trouver des compatriotes pouvant les aider. Petit à petit une importante colonie se développa autour du marché.

41 Dans les années quatre-vingts, plusieurs activités économiques à l'initiative des immigrants se développèrent : les Portugais se spécialisèrent dans la vente de produits agricoles, de mercerie et de tissus; les Allemands dans celle des machines et instruments métalliques; les Italiens dans les blanchisseries, boulangeries, salons de coiffure et ateliers de couture ainsi que dans la vente de matériel de construction.

Dans l'almanach de 1893, on trouvera les références de ces commerces tenus par des Libanais ou des Syriens (six merceries et une épicerie). Knowlton considère qu'à cette époque, plus de 90 \% des colporteurs de la ville de São Paulo appartenaient à ce groupe. Ils avaient réussi à supplanter les colporteurs italiens. En 1901, on recensait plus de 500 établissements appartenant à des Libanais ou des Syriens. Les registres des impôts du commerce de 1907 indiquent que sur les 315 entreprises syriennes ou libanaises de São Paulo, 219 étaient des magasins de tissu au détail ou des magasins de tissu et mercerie (Knowlton, 1961).

Durant les années vingt, ils commencèrent à occuper des positions plus favorables dans le commerce en gros de tissu, les merceries et l'industrie de la confection grâce aux marchés conquis pendant la guerre, lors de la suspension des importations. Dans ce secteur, le commerce était aussi dominé par les entreprises portugaises. Les registres des impôts de 1907 indiquaient onze magasins de tissu en gros appartenant à des Libanais, nombre sûrement inférieur à la réalité mais dû à l'omission de déclaration, pratique alors très répandue. À mesure que les affaires de la colonie se multipliaient avec de nouveaux magasins de détail, quelques importantes entreprises de vente en gros s'installèrent au centre ville pour les approvisionner. Le recensement de 1920 confirme que parmi les 91 établissements industriels libanais et syriens enregistrés, 65 se trouvaient dans le secteur de la confection, 12 dans le secteur textile et 8 d'entre eux dans la branche des mailles et chaussettes. Ceux qui choisirent cette voie ne le regrettèrent pas. Les années vingt se montrèrent exubérantes pour l'industrie textile au Brésil. Après la guerre, les importations de machines furent importantes, grâce notamment à des conditions de paiement facilitées par les fabricants anglais avides de reconstituer leurs flux commerciaux (Stein, 1979).

44 La crise de la fin des années vingt obligea plusieurs commerçants à repartir à zéro. Comme les entrepreneurs syro-libanais prenaient des risques maximum, un grand nombre fit faillite. Cependant, bien que des fortunes aient été perdues du jour au lendemain, les membres de la colonie conservèrent leur rang économique. En 1934, 
parmi les groupes d'immigrants les plus importants de São Paulo, les Libanais et Syriens furent ceux, en moyenne, dont les entreprises se comportèrent le mieux (Estatística, 1934).

Durant trente ou quarante ans, les Syriens et les Libanais consolidèrent leurs affaires, créant un monopole du commerce de tissu au détail et de la petite industrie, occupant la position la plus importante dans le commerce en gros. Leurs investissements représentent la moitié du capital placé dans l'industrie textile de São Paulo durant les années quarante. Il est aussi significatif que, comme l'a montré José Carlos Durand (1985), le capital syrien et libanais se soit transféré du commerce de détail au commerce en gros, surtout dans l'industrie. Il est évident que la colonie affermit ainsi son pouvoir économique, s'intéressant maintenant à des positions plus importantes dans les secteurs commerciaux et industriels. Ainsi s'établit un secteur totalement intégré verticalement, où industriels et vendeurs en gros répondaient aux nécessités d'un grand réseau de vendeurs de détail et de colporteurs, tous de la même origine.

Éléments d'une trajectoire réussie

Un bilan de l'histoire de la réussite de la colonie syrienne et libanaise, en ce qui concerne son ascension économique, ne peut oublier de souligner trois éléments importants.

Premièrement, les Libanais et les Syriens présentaient un profil de distribution spatiale singulier. À la différence des autres groupes, ils réunissaient simultanément deux caractéristiques: ils étaient bien répartis dans les différentes régions du territoire brésilien et, en même temps, très présents dans les zones urbaines. Cette conjonction unique est caractéristique de cette colonie qui détenait des commerces dans tout le Brésil, ce qui faisait dire que " on trouvait un Turc avec un petit commerce même dans les plus petits villages » (Curban, 1991).

Le deuxième élément concerne les rapports de complémentarité et d'entraide établis au sein de la colonie. Ceux-ci se manifestèrent dans un grand nombre de comportements, allant de l'accueil des nouveaux arrivants, aux relations établies entre industriels et grands commerçants. Entre les deux, on retrouve un ensemble de mécanismes facilitant le crédit, la fourniture de marchandises, ce qui, sur toute la chaîne commerciale, forma une base importante de ces activités économiques. Bien que leurs établissements soient concurrents, il est certain que les entrepreneurs syriens et libanais bénéficièrent des effets de cette concentration. Au début, la concurrence avec d'autres nationalités avait été dure et dénuée de toute confiance dans les affaires (Safady, 1949). Entre membres du groupe, les conflits pouvaient être résolus par un ami ou encore par les chefs ou patriarches de la colonie. De plus, travaillant dans une époque de création d'un marché de travail urbain, alimenté aussi bien par les populations de la campagne que par celles qui avaient quitté les villes ouvrières fermées des grandes entreprises, tous ceux qui cherchèrent à s'installer soit comme colporteur, soit comme commerçant, trouvèrent un espace.

49 Enfin, il faut souligner le processus continu de ré-alimentation favorisé par l'arrivée de parents et compatriotes. Il n'existe pas au Brésil de données précises à ce sujet, mais tout indique que cet effet de "chaîne " fut caractéristique des immigrants syriens et libanais. Sans cela, ils se seraient probablement limités à la capacité de travail du noyau familial. Cet effet de chaîne leur a permis de se différencier et de donner à leurs compatriotes, déjà longuement établis, la possibilité d'atteindre des positions 
stratégiques dans le commerce en gros ou dans l'industrie liée à ce secteur. Ainsi, s'édifièrent de grandes fortunes.

La plupart des immigrants ne sont pas arrivée seuls et abandonnés. Ils avaient pris la décision d'immigrer à la suite d'informations raisonnablement précises sur les lieux d'accueil, la façon de trouver un travail et surtout le genre de travail qu'offrait cette nouvelle société. Dès le début, ils avaient une notion très claire du contexte, transmise par ceux qui les avaient précédés : où il fallait commencer, le créneau où l'on pouvait s'établir avec succès, les réseaux de compatriotes; tout cela permettait d'aider le nouvel arrivant à s'intégrer dans la société en lui donnant un emploi, une formation. Afin d'attirer une nouvelle clientèle, et en raison des caractéristiques de leurs affaires, les Libanais et les Syriens voulant s'implanter devaient faire preuve d'une grande sociabilité, établir des contacts durables avec ceux qui vivaient dans la même rue, le même quartier, le même village.

51 Par ailleurs, en plus des liens de concitoyenneté, les rapports familiaux étaient très denses à l'intérieur de la colonie. Entre les Libanais et les Syriens, l'économie familiale s'est maintenue et développée car du travail de toute la famille dépendait la réussite des affaires. Organiser la famille fut l'objectif de ces immigrants pour s'installer dans cette nouvelle société.

"Souvent un colporteur syrien ou libanais de la rue 25 de Março repérait une rue ou une place qui semblait particulièrement favorable aux affaires. Au fil des jours, les gens du voisinage commençaient à le connaître comme le colporteur qui passait tous les jours devant leur porte et ils lui accordaient leur préférence. Après avoir beaucoup épargné, le colporteur cherchait une boutique disponible dans un bon endroit, près de ses clients et installait une petite mercerie qui vendait aussi des tissus et des vêtements. Il agrandissait son magasin au fur et mesure de ses gains et faisait venir sa famille et des compatriotes. Plusieurs ont fait cela. Ils accumulaient un capital et, à leur tour, installaient leur propre affaire dans la même zone. Avec le temps, chaque district et quartier de São Paulo parvint à avoir un noyau de Libanais ou de Syriens originaires du même village ou de la même ville.» (Knowlton, 1961 : 118)

Deffontaines (1936: 29) avait déjà observé que «le commerce de colporteurs représentait un progrès significatif pour une économie essentiellement fermée. Avec leur esprit commerçant, ils créèrent une grande affaire et participèrent activement à la naissance de la grande industrie. » Des années plus tard, Knowlton (1961: 138) sera impressionné par ces qualités commerciales. "Ils n'avaient pas de prix fixe, ils vendaient au prix qu'ils croyaient juste et ils vivaient avec très peu de moyens. Si le client n'avait pas d'argent pour payer, le colporteur acceptait en troc du caoutchouc, du bétail, du café, de l'or ou n'importe quel autre produit. Les Libanais étaient aussi disposés à accorder un crédit durant un an. Grâce à leur flexibilité et à leur acceptation du risque, ils n'avaient pas de concurrents parmi les autres catégories d'immigrants ».

Ellis Jr. (1934 : 198) ajoute que « le Syrien ne mit pas longtemps à surpasser le Portugais urbain avec son commerce de quincaillerie; le Portugais travailleur et honnête, mais traditionaliste, conservateur et ennemi du progrès ». Knowlton (1961:137) a constaté que les Syro-Libanais réussirent à expulser les Italiens (en majorité Calabrais) du métier de colporteur de tissu et de mercerie, en adoptant le système de vente à crédit et de troc. Au début du siècle, ces Italiens tentèrent de convaincre leurs fournisseurs de boycotter les colporteurs libanais. Mais cette tentative échoua.

Les Libanais et les Syriens entreprirent, en pionniers, une véritable révolution des pratiques commerciales; ils donnèrent un nouveau souffle au commerce, redéfinirent 
les conditions de réalisation des profits pour tout le secteur en adoptant une politique de ventes à crédit, en cherchant une compensation à la réduction de leur marge unitaire par l'augmentation des ventes et par une plus grande rotation des stocks, en inaugurant les soldes, en réinvestissant leurs gains dans leur propre affaire et, enfin, en accordant plus d'attention aux besoins et aux conditions des consommateurs. Il n'est donc pas exagéré d'affirmer qu'ils sont au Brésil les «inventeurs» du commerce populaire.

55 L'histoire des Libanais et des Syriens souligne que ces immigrants ont mis en place des réseaux d'emplois, des établissements réalisant des affaires préférentielles entre compatriotes et membres de la famille, et se sont constitués, dans tout le Brésil, de vrais fiefs. À la longue, plusieurs ont délaissé la condition de colporteur ou de petit commerçant pour prendre la tête d'entreprises industrielles ou de réseaux de distribution en gros. Mais, quelques soient leur ascension sociale ou leurs origines, ils doivent se rappeler des opportunités antérieures, rendues possibles grâce à la collaboration de la famille, des relations de parenté et de concitoyenneté offertes par la colonie et mobilisées en faveur des réussites individuelles.

Identités et images

56 L'évolution de l'image attribuée à la colonie constitue, sans doute, un des thèmes intéressants de l'histoire des Libanais, des Syriens et de leurs descendants au Brésil. De leur point de vue, les Brésiliens étaient considérés comme indolents, peu travailleurs et, pour ces raisons, les mariages mixtes devaient être évités. De plus, les contrôleurs fiscaux, ennemis par excellence des colporteurs, étaient tous Brésiliens.

Aux yeux de la société brésilienne, tout le groupe était associé, avant tout, à l'image du commerçant. Des années de colportage, la manière particulière de marchander pour réaliser des affaires, son aspect et le fort accent caractéristique fixèrent un stéréotype définissant la façon dont ils gagnaient leur vie. «Par les voies publiques, transportant, comme des boufs, au soleil, transpirants et lents, la cantine pleine de quincailleries ou portant en bandoulière, comme les plats d'une balance, des paniers avec des légumes ou des fruits, ils partent, collaborateurs forcés de notre progrès, déclamant avec les difficultés de la langue, leur attirante marchandise. On les voit, - tête ovale, regard pénétrant et subtil, moustaches longues et noires, mains et bras pleins de tatouages machiavéliques, - derrière le comptoir du magasin, exhalant l'odeur d'empois de tissu neuf, insister auprès du client, tenant le mètre, pendant les "bachinchas » pour, deux heures après, vendre pour 1,350 \$ le mètre de tissu, dont il avait demandé au départ 5 \$; la négociation avait été lente, en dose homéopathique, avec des grimaces, des plaisanteries, des justifications interminables. Les affaires sont, pour eux, à la mesure de la patience ou de la bonne humeur du client » (Moura, 1954 : 138).

Déjà, pendant les premières décennies $\mathrm{du} \mathrm{xx}^{\mathrm{e}}$ siècle, on trouve les principaux éléments qui composeront l'image la plus commune et la plus populaire des Syriens et des Libanais : l'aspect et la langue caractéristiques, l'identification avec le colportage et la façon particulière de marchander pour réaliser une affaire. Il est compréhensible qu'en se consacrant en nombre à une activité définitivement liée au commerce de biens de consommation, les Syriens et les Libanais aient pour cela reçu une marque, une appréciation fixant leur image dans la société. À cette image s'est apposée une désignation : turcs.

59 Jusqu'à la fin de la Première Guerre mondiale, presque tous les immigrants de la colonie sont venus au Brésil avec un passeport turc, ce qui, à leur grand déplaisir, les identifiait à leur dominateur. Le phénomène n'était pas uniquement restreint au Brésil: en 
Argentine (et les autres pays d'Amérique Latine), Syriens et Libanais furent et sont encore couramment appelés "turcs». C'est un motif de plaintes courant et un sujet dont traite la quasi-totalité des livres écrits par la colonie.

Au qualificatif de « turc » employé amicalement ou péjorativement, fut associée aussi la capacité de négocier n'importe quelle affaire. Hajjar (1985) dit que l'expression la plus douloureuse pour les Arabes au Brésil était " turc à crédit ", qui figurait même dans les dictionnaires. Plus tard, ce surnom sera étendu aux Juifs, qui étaient aussi colporteurs (Lesser, 1989). Une image si clairement définie a contribué à ce que la dénomination de " turc » les accompagne jusqu'à aujourd'hui et soit toujours utilisée pour les désigner.

Dans le roman "Gabriela Cravo e Canela", de Jorge Amado (1975), le plus grand écrivain brésilien contemporain, un des protagonistes s'appelle Nacib6.

«Plusieurs le traitaient d'Arabe et de Turc, c'est vrai. Plus exactement ses meilleurs amis et ils le faisaient d'une façon affectueuse et dans l'intimité. Il n'aimait pas qu'on l'appelle le Turc; il chassait irrité ce surnom, parfois il arrivait à se fâcher :

- Turc, c'est ta mère!

- Mais Nacib....

- Tout ce que tu veux, sauf Turc. Brésilien - il tapait avec sa main énorme sur sa poitrine pleine de poils - fils de Syrien, par la grâce de Dieu.

- Arabe, Turc, Syrien c'est tout la même chose.

- La même chose, cocu ! C'est de l'ignorance de ta part. C'est ne pas connaître l'histoire et la géographie. Les Turcs sont des bandits, la race la plus infortunée qui existe. Il n'y a pas d'insulte plus grave pour un Syrien que d'être appelé de Turc.

- Voyons, Nacib, ne te fâches pas. Je n'ai pas voulu t'offenser. C'est que ces choses de l'étranger pour nous c'est tout pareil... "

62 Comme dans l'histoire de Nacib, le reste de la société brésilienne n'avait pas de critères pour distinguer Libanais et Syriens et leurs origines. Ils furent rassemblés dans une catégorie moins précise et plus générale. Aux yeux d'une personne extérieure à la colonie, les identités des villages et des provinces d'origine, si importantes pour chacune des deux collectivités, n'existaient pas.

63 À l'image familiale de commerçant s'en superposèrent d'autres: les Turcs se mélangeaient très peu, parlaient une langue incompréhensible et se mariaient entre eux, faisant venir souvent leur future épouse de leur pays d'origine.

De plus, il est certain que se consacrer au commerce - une activité qui, en général, éveille le soupçon de gains faciles - provoque la formation de préjugés. Pour le client, cependant, la commodité offerte par le fait d'acheter des marchandises à sa porte, soit en ville soit en zone rurale, de payer avec des délais, surmonta toutes les appréciations négatives. Les plaintes et les plus grands préjugés par rapport aux Turcs vinrent surtout de ceux qui en ont subi la concurrence, les commerçants d'autres nationalités établis dans des magasins relayés par les autorités locales. Ainsi, dans l'État de São Paulo, un membre de la municipalité de São Carlos, lors d'une séance du conseil, soulignait avec une verve enflammée le fait que :

"... aucun des Turcs arrivés au Brésil n'avait tenu la houe pour laisser dix pieds de café plantés pour le bien du pays. Qu'est-ce qu'il fallait attendre alors de l'immigration turque? ... Le travail de ces pantalons larges est seulement de marchander, payant 10000 \$ le permis de vendre des marchandises de toutes sortes (comme commerçants), trompant les heureux idiots dans la valeur des billets, en rendant la monnaie et après avoir gagné beaucoup d'argent, quittant le Brésil sans laisser aucun gain, parce qu'ils ne mangent même pas, et cet 
argent ne revient plus par ici parce que le Brésil n'a pas de commerce avec la Turquie, ni

avec le sultan, ni avec ses quatre cents épouses » Damiano, 1996 : 26). fondateur de ce groupe et fut rendu célèbre en prose et en vers par les intellectuels de la colonie (Safady, s/d ; Féres, 1970). En effet, le personnage du colporteur a constitué la seule base possible d'identité collective d'une colonie fragmentée entre différentes religions et régions d'origine. Plus que cela, des qualités comme celles le qualifiant de travailleur, frugal et persévérant furent, de façon insistante, réaffirmées et montrées comme des exemples de conduite. Sa capacité d'adaptation impressionna et fut à l'origine d'histoires où la fable et la réalité se confondaient, comme l'épisode raconté par Bastani (1945) dans son livre « Memórias de um mascate » . L'auteur raconte l'histoire du Libanais Kalil, que son compagnon Miguel croyait mort, est qui fut retrouvé douze ans après chef d'une tribu d'indiens en Amazonie.

Différences et intégration

$67 \mathrm{Si}$, aux yeux de la société brésilienne, il y avait des raisons de considérer les Libanais et les Syriens comme un groupe uniforme, une analyse plus attentive met en valeur son caractère stratifié, composé de fractions possédant, en général, des ressources et des aptitudes différentes, ainsi que des orientations divergentes. Une complexe hiérarchie de statut et de pouvoir s'établit lentement au sein de la colonie; elle résulta non seulement de filiations religieuses, d'origines géographiques et de situations politiques survenues dans leur terre d'origine, mais, surtout, de performances économiques différentes entre familles dans la nouvelle société. Le capital accumulé était essentiellement fonction de l'ancienneté d'arrivée au Brésil. Les grandes fortunes commerciales et industrielles de la colonie durant les années quarante et cinquante furent édifiées par les familles qui commencèrent, en premier lieu, à parcourir le chemin de colporteur, vendeur au détail, puis vendeur en gros et industriel. Parmi les Libanais, ce sera le cas de la famille Jafet.

Les lieux d'habitation des membres de la colonie constituèrent peut-être l'indice le plus visible des différences ; pour l'immigrant récemment arrivé, le logement typique était l'habitation commune des pauvres. Pour celui qui avait déjà installé un magasin, la participation de la famille dans l'affaire exigeait qu'elle vive dans le même immeuble, soit à l'étage supérieur soit derrière le magasin. Quelques années plus tard, les familles les plus prospères déménagèrent pour habiter d'autres quartiers (Toledo, 1987 ; Barro et Bacelli s/d ; Knowlton, 1961).

La fin de la Première Guerre mondiale donna un nouvel essor à l'immigration et le parcours typique des immigrants de cette colonie soit comme colporteurs, soit comme petits vendeurs au détail repris. Les pionniers, certains possédant d'énormes fortunes, leur montraient le chemin, commencé vingt ou trente ans auparavant, qui les avaient menés au succès. Pour les nouveaux arrivants, le problème était précisément ce déphasage dans le temps qui se manifestait par un nombre moins important 
d'occasions et par une compétition plus forte, au sein de leur propre groupe mais aussi en dehors (en particulier avec les Juifs).

70 La grande majorité de ceux, arrivés à cette date, n’accédera jamais à la condition de petit propriétaire d'un magasin. Immigrer en Amérique et y faire fortune ne fut qu'un rêve pour ces derniers arrivés. Pour ceux qui, en général, avaient une famille ou des compatriotes établis depuis longtemps, il était encore possible d'accéder à une certaine prospérité. Mais la plupart durent partir faire fortune dans des endroits éloignés des centres des villes ou des capitales, construisant la réputation et la popularité des « Turcs » dans tout le Brésil.

71 "Miguel Jorge descendit à ce port dont le nom est si long: Santa Maria de Belém do Grão Pará. Tout de suite on l'avisa : «Ici, il y a déjà suffisamment de "Turcs" (...) Après plus de vingt jours, il est arrivé dans une autre ville, un peu plus grande, Manaus. Et, de nouveau, il a vu qu'il y avait un surplus de compatriotes, quelques uns travaillant pour d'autres, plus prospères. Alors, on lui donna quelques marchandises, on lui apprit les noms, les prix et on lui enseigna la direction à suivre: "Vas de ce côté, en amont. Toujours en amont. Entre là et, de là, va vers une autre rivière. Tu regardes les rives, tu regardes les gens, si tu en vois un qui n'est pas nu, c'est un client. Quand la personne n'a pas d'argent tu fais du troc. Si c'est du caoutchouc, tu le coupes en quatre, pour être sûr qu'il n'y ait pas de bois dedans. Quand tu peux, reviens pour payer ce que tu as emporté. » (Farhat, 1987 : 54)

72 En plus des décalages de revenus les différences internes à la colonie se manifestèrent dans les associations d'entraide, notamment les associations religieuses. Les premiers responsables de la colonie bénéficièrent de la reconnaissance de leur communauté car ils établirent et financèrent des institutions mutuelles ou religieuses. Ironiquement, cependant, à mesure que ces organisations s'institutionnalisaient, les notions de statut et de pouvoir s'établirent entre les membres de la communauté. Les figures les plus en vue rivalisèrent de prestige par le biais de ces associations, les finançant avec générosité, leur donnant ainsi les moyens de fonctionner.

73 Les Libanais et les Syriens constituaient un groupe bien défini par des identités ethniques, religieuses et régionales; les critères et les raisons justifiant la création de ces institutions, en général, tenaient compte d'une de ces dimensions, présentes dans la plupart des institutions fondées par la colonie. L'insertion ethnique, religieuse et régionale si décisive dans leur pays natal, ne permettrait pas, lors de l'arrivée au Brésil, d'oublier les tensions antérieures. La compétition entre les responsables mobilisait des références importantes et décisives en termes d'identités, et elle finit par conditionner la sociabilité de la colonie, en particulier autour d'innombrables associations de bienfaisance, écoles, clubs, entités philanthropiques, journaux qui, rivalisant, se multiplièrent.

$74 \mathrm{Au}$ fil du temps, la tendance générale de ces organisations fut de constituer un groupe d'associés, défini davantage par leurs filiations de classe que par leurs origines communes. Plusieurs clubs existent encore aujourd'hui et ne maintiennent pas de critères rigides de filiation. Alors que dans les années trente, le nombre des immigrants diminuait, une part importante de ces associations, qui se consacraient surtout à assister des nouveaux arrivants, n'eut pas de problèmes à se transformer en entités à des fins sociales et culturelles ou en clubs et cercles dépassant ainsi les premiers objectifs. Ces associations réduirent leurs aspects mutualistes et se tournèrent vers la 
classe moyenne où prédominaient les compétitions sportives, les bals de débutants et les soirées littéraires.

Pour les nouveaux riches de la colonie et leurs descendants de la première et de la deuxième génération, les clubs furent le lieu des mariages par excellence. Le schéma « rentrer au pays d'origine pour se marier / se marier avec un cousin / se marier au club ", associé à des générations successives révèle que, pour les riches, un mariage avec un Brésilien ou un autre étranger fut plus lent à se réaliser que pour le reste de la colonie. C'est seulement à partir de la troisième génération que l'intégration se fit d'une façon exemplaire.

L'incorporation de la cuisine arabe à la cuisine nationale illustre la forte interaction entre les deux cultures. Il s'agit d'un phénomène singulier, qui a atteint des proportions beaucoup plus importantes que dans n'importe quel autre pays accueillant des contingents d'immigrants libanais, comme les États-Unis ou l'Argentine. Bien que plusieurs fois modifiées, certaines recettes très répandues par les immigrants font parties aujourd'hui de la cuisine brésilienne, comme le quibe, la esfiha, le taboulé, le yaourt, le babaganouche, le pain syrien et les lentilles. De plus, des restaurants spécialisés en cuisine arabe (ou inspirés de la cuisine arabe) proliférèrent dans tout le Brésil et il n'existe pas de guide gastronomique sans une section correspondante ni un shopping center sans un restaurant arabe.

Médecins et hommes politiques

Le comptoir commercial était loin de représenter le point final de la trajectoire d'ascension socio-économique. Parallèlement à l'expansion de leurs affaires dans les secteurs commerciaux et industriels, l'entrée des descendants de Libanais et de Syriens dans les professions libérales (droit, médecine et génie civil) constitua une alternative poursuivie avec succès par d'importantes fractions de la colonie. À São Paulo, une enquête faite parmi les élèves d'origine étrangère ayant terminé leurs études à la Faculté de Droit, de Médecine et de l'École Polytechnique entre 1880 et 1950 confirma l'entrée importante, à partir des années trente, de descendants de Libanais et Syriens dans ces écoles formant l'élite des membres des professions libérales (Truzzi, 1992). En effet, la rapidité de l'ascension économique a rendu possible l'envoi dans des écoles de haut niveau des enfants d'immigrants. D'après des enquêtes réalisées dans les écoles, le nombre de futurs diplômés, dont les parents commencèrent comme colporteurs, est remarquable. La réussite commerciale de leurs parents leur a permis de s'intégrer dans le secteur des professions libérales, en pleine formation dans les années trente. Certes, toute la communauté ne bénéficia pas de cette possibilité, mais certains surent mieux profiter que d'autres de cette opportunité. Plusieurs immigrants ne voulaient pas que leurs enfants connaissent les difficultés d'une activité dont le début avait été si dur et les voir avec un diplôme d'enseignement supérieur représentait une compensation à une vie de sacrifices.

78 La trajectoire des familles de ceux qui obtinrent leur diplôme universitaire dans des écoles d'élites donne lieu à un ensemble varié d'histoires intéressantes et significatives. Le plus remarquable est que, en une génération à peine le saut entre l'activité de colporteur et l'obtention d'un diplôme supérieur fut réalisé.

Certains immigrants arrivèrent, dans les années vingt, avec une formation en médecine de l'Université Américaine de Beyrouth, et en ayant suivi des stages en Europe ou aux États-Unis. À São Paulo, leurs descendants - surtout du sexe masculin - choisirent en majorité la médecine. Ils créèrent en 1922 l'Association des Anciens Élèves de 
l'Université Américaine de Beyrouth. Parmi les cent médecins membres de cette association, soixante-dix résidaient à São Paulo (Lacaz, 1982). Ce transfert au Brésil de professionnels déjà formés a sûrement stimulé les vocations dans la colonie. Des compatriotes médecins, déjà établis, ont certainement facilité l'insertion professionnelle des plus jeunes en leur transmettant les règles du métier et une clientèle afin de développer cette carrière. Dans la logique de rapports préférentiels entre compatriotes, les médecins, comme les autres membres des professions libérales eurent beaucoup de clients parmi les membres de la colonie.

Grâce à cette arrivée remarquée des descendants de Libanais dans le secteur des professions libérales, soit en conquérant des positions déjà existantes, soit en créant de nouveaux espaces d'insertion et en se valorisant socialement (comme dans le cas de l'Hôpital Syro-Libanais de São Paulo), l'image de la colonie, reconnue auparavant pour ses seules qualités commerciales, se diversifie. L'extrême importance de cette ascension réside dans le fait que les enfants, en choisissant d'exercer des professions libérales, apparaissent comme "nettoyant le sang » du groupe; ils exerçaient des professions d'une valeur universelle et d'un savoir plus légitime que le commerce, leur conférant une reconnaissance de la société dans son ensemble. Sans ce pas préalable, la colonie n'aurait jamais pu, à partir de la fin des années quarante, recueillir les fruits de cet investissement éducatif dans le domaine de la politique.

$81 \mathrm{Au}$ début de l'immigration, pour la majorité des immigrants d'origine syrienne ou libanaise au Brésil, la politique représentait une activité très lointaine. La majorité de ceux qui, après la Deuxième Guerre mondiale, s'est lancée dans la carrière politique était issue de familles dont les parents avaient commencé comme colporteur à peine une génération auparavant. Leurs enfants - plusieurs d'entre eux avaient suivi des études supérieures dans des écoles de prestige - s'essayaient à une carrière politique afin de continuer le remarquable processus d'ascension sociale précédemment entrepris. Pour cela, le soutien de la colonie, surtout au début, était très important. Sur ce fondement initial et cette base électorale se construisit la plupart des carrières politiques.

Les premiers de la colonie à entrer en politique, après 1945, sortirent diplômés d'écoles prestigieuses. À São Paulo, les bancs de la Faculté de Droit seront une pépinière d'hommes politiques. À partir des années soixante, l'entrée de descendants de Libanais et Syriens en politique se généralisa. Un des éléments de leur sur-représentation, qui n'est pas proportionnelle à son nombre, réside dans la combinaison particulière de deux facteurs. D'abord, la dispersion géographique de la colonie qui contribua à répandre l'idée que « aujourd'hui, il existe un compatriote dans n'importe quel trou perdu du pays» selon l'expression d'un interviewé. Ce premier facteur fut très rapidement un élément qui fit émerger des responsables locaux.

Il convient cependant de relativiser l'influence du vote de la colonie. Une interprétation correcte reviendrait à attribuer l'entrée en politique au profond enracinement social du groupe qui, sans perdre son identité, sut se fixer de manière irréversible dans le tissu social. Et il n'existe pas de meilleur exemple que celui des politiciens d'origine libanaise contrôlant les clubs de football de São Paulo, le «jogo do bicho " (tombola dont les numéros sont désignés par des noms d'animaux) et les écoles de samba de Rio de Janeiro. Cette forte représentation de la colonie dans le domaine politique fut constatée dans tous les Etats de la fédération. En 1987, une publication destinée à servir de référence à la communauté libanaise au Brésil, présenta avec un 
orgueil non feint, le grand nombre de membres du groupe parlementaire Brésil-Liban : 33 députés nationaux, 7 sénateurs et 2 gouverneurs de province (Khodr, 1987).

En outre, cet enracinement social des membres de la colonie, grâce à l'entrée de contingents significatifs en politique, a été facilité par les caractéristiques du « milieu récepteur »: une société hétérogène et relativement ouverte, en expansion économique, formée de différentes strates sociales peu consolidées et qui mettait sur le même rang des Brésiliens de plusieurs générations, des immigrants de plusieurs origines, des enfants d'immigrants, etc. Une comparaison avec la trajectoire des descendants de Libanais dans d'autres pays comme les États-Unis ou l'Argentine, par exemple, montre bien toutes les facilités d'intégration que, d'une manière générale, la société brésilienne offrait (Truzzi, 1997b). Stefan Zweig (1941) écrivait, en arrivant au Brésil : "Tout de suite, en arrivant dans ce pays, la première surprise, qui, heureusement, se renouvelle tous les jours, est la manière chaleureuse et non fanatique dont les êtres humains vivent dans ce gigantesque territoire. Sans le vouloir, on respire de nouveau, on se sent bien pour avoir quitté cet air maléfique de haine entre races et classes ennemies et se trouver dans une atmosphère humaine."

La gratitude de nombreux immigrants libanais et syriens envers leur nouvelle patrie ne fut pas longue à s'exprimer. En 1922, quand le Brésil fêta le centenaire de son indépendance, la colonie libanaise de São Paulo, composée à l'époque d'immigrants récemment arrivés, décida d'offrir un monument en bronze, à la mémoire de cet événement. Sur sa base, un poème, sélectionné par concours, dont le contenu proclamait :

Si l'on coupait tous les cèdres du Liban, et le cèdre est notre source d'inspiration, et qu'avec eux on érigeait ici un temple dont les tours traverseraient les nuages; si on enlevait de Baalbeck et de Palmyre les vestiges de notre glorieux passé ; si on arrachait de Damas la tombe de Saladin, et de Jérusalem le Sépulcre du rédempteur des hommes;

si on apportait tous ces trésors à cette grande nation indépendante et à ses glorieux enfants

on verrait que, même ainsi on ne serait pas suffisamment reconnaissant envers le Brésil et les Brésiliens.

Pour ceux qui arrivèrent très jeunes, comme Nacib, l'adhésion aux valeurs du nouveau pays fut encore plus incontestable :

"-Dans le pays de mon père... - c'est ainsi que les histoires commençaient dans les nuits de longues conversations, quand il ne restait que des amis dans les bars. Parce que son pays était Ilhéus, ville gaie en face de la mer, des plantations de cacao, cette zone très fertile où il grandit. Son père et ses oncles suivant l'exemple des Aschcar, arrivèrent en premier, laissant leurs familles. Son père était arrivé, avec sa mère et sa sœur aînée, âgée de six ans. Nacib, n'avait pas encore quatre ans. Il avait des souvenirs très vagues du voyage en troisième classe, du débarquement à Bahia où son père l'attendait. Ensuite, de l'arrivée à Ilhéus et du voyage en canot, puisque à l'époque il n'existait même pas de quai. Il n'a aucun souvenir de la Syrie, comme il ne lui restait aucun souvenir de son pays natal, il s'était fait brésilien et citoyen d'Ilhéus. Pour Nacib, c'était comme s'il était né au moment de son arrivée en bateau à Bahia, quand son père en larmes l'embrassa » (Amado, 1975 : 45).

Aujourd'hui, plus d'un siècle après l'arrivée des premiers immigrants, le bilan de leur trajectoire et de leur vie est constitué de récits émouvants recueillis dans les interviews réalisées auprès des plus âgés, capables de regarder en arrière et conscients des difficultés vécues et du chemin parcouru. «Dans la vie brésilienne, on acquiert depuis 
l'enfance une tolérance qui là-bas n'existe pas... Je suis heureux de ma vie ici, j'ai confiance en ce pays, ici c'est ma terre » (Greiber et alli, 1999).

Conclusion

déplacement vers le Brésil doit être vu comme un phénomène social encourageant des groupes, articulés en réseaux, à émigrer pour sortir leurs familles de situations défavorables. Au Brésil, ces réseaux familiaux et de compatriotes sont demeurés très actifs, en accordant accueil et travail pour les nouveaux arrivés.

89 dispersion géographique et leur insertion urbaine prédominante, j'ai défini les étapes principales de leur remarquable mobilité socio-économique: colporteur / petit commerçant / grossiste / industriel ; un itinéraire qui n'a pas été suivi par tous, mais qui a conformé le domaine du groupe ethnique sur un secteur industriel et commercial important. Au long de ce parcours, de nouvelles identités ont été bâties, en réponse aux images du groupe devant la société et du propre procès de différentiation interne à l'ethnie. Concernant la deuxième génération, le choix est de placer les enfants sur le marché des professions libérales, essentiellement comme médecins; choix qui à son tour facilitera l'entrée sur la scène politique.

Les trajectoires des immigrants libanais et syriens dans la société brésilienne, et particulièrement à São Paulo, illustrent de façon exemplaire les opportunités offertes par une société en effervescence, devenant de plus en plus complexe. Ces immigrants et leurs descendants ont bouleversé les structures sociales polarisées d'une société agraire en décomposition, en inaugurant un nouvel ensemble de positions intermédiaires à l'intérieur du tissu social.

\section{BIBLIOGRAPHIE}

AMADO Jorge (1975) Gabriela Cravo e Canela, São Paulo, Círculo do Livro.

ANDRADE Moacir (1985a) Manaus: Ruas, Fachadas e Varandas, Manaus, Humberto Calderaro.

ANDRADE Moacir (1985b) Tipos e veículos de transportes fluviais do Amazonas, Manaus, Imprensa Oficial.

ARAUJO Oscar (1940) Enquistamentos Étnicos, Revista do Arquivo Municipal de São Paulo, n. 6.

BARRO Máximo et BACELLI Roney (s/d) Ipiranga, (série História dos bairros de São Paulo), São Paulo, Prefeitura Municipal / Secretaria Municipal da Cultura.

BASTANI Tanus (1945) O Líbano e os libaneses no Brasil. Rio de Janeiro, Artes Gráficas.

BENCHIMOL Samuel (1985) Grupos Culturais na Formação da Amazônia Brasileira e Tropical, (mimeo), Manaus. 
BESTENE Jorge (1992) Formas de Asociacionismo entre los Sirio-Libaneses en Buenos Aires (1900-1950), in Fernando Devoto e Eduardo Miguez Éds., Asociacionismo, Trabajo e Identidad Etnica. Buenos Aires, CEMLA-CSER-IEHS.

CURBAN Guilherme (1990) Interview.

DAMIANO Otávio (1996) Imprensa São Carlense. 1976-1995. São Carlos.

DEFFONTAINES Pierre (1936) Mascates ou pequenos negociantes ambulantes do Brasil, Geografia, $2: 1$.

DIEGUES Jr. Manuel (1951) Dois grupos étnicos-culturais no Brasil: italianos e sírio-libaneses, Jornal do Commércio, 4 de outubro.

DUOUN Taufik (1944) A emigração sírio-libanesa às terras da promissão, São Paulo, Tipografia Ed. Árabe.

DURAND José Carlos (1985) Formação de pequeno empresariado têxtil em São Paulo (1880-1950), in Henrique Rattner Éd., Pequena empresa - o comportamento empresarial na acumulação e na luta pela sobrevivência, São Paulo, Brasiliense.

ELLIS Jr. Alfredo (1934) Populações Paulistas, São Paulo, Companhia Editorial Nacional. Estatística Industrial do Estado de São Paulo, 1934.

FARHAT Emil (1987) Dinheiro na estrada : uma saga de imigrantes, São Paulo, T. A. Queiroz.

FÉRES Assis (1970) O mascate, São Paulo, Laiazul.

GREIBER Betty Loeb et alii (1999) Memórias da Imigração - libaneses e sírios em São Paulo. Discursos Editorial, São Paulo.

HAJJAR Claude (1985) Imigração Árabe : 100 anos de reflexão, São Paulo, Ícone.

HITTI Philip (1951) Hystory of Syria, including Lebanon and Paletine, London, Macmillan.

HITTI Philip (1967), Lebanon in History, London, Macmillan.

HITTI Philip (1924), The Syrians in America, New York, George H. Doran.

KHODR Hermat (1987) O Libanês no Brasil.

KNOWLTON Clark (1961) Sírios e Libaneses : mobilidade social e espacial, São Paulo, Anhambi.

KURBAN Taufik (1933) Os Sírios e Libaneses no Brasil, São Paulo, Sociedade Impressora Paulista Ltda.

LACAZ Carlos (1982) Médicos sírios e libaneses do passado - trajetória em busca de uma nova pátria, São Paulo, Almed.

LESSER Jeffrey (1989) Pawns of the Powerful : Jewish Immigration to Brazil (1904-1945), New York University (PhD dissertation), New York.

MILLER Deborah (1981) Middle Easterners : Syrians, Lebanese, Armenians, Egyptians, Iranians, Palestinians, Turks, Afghans, in They Chose Minnesota : a Survey of the Stateds Ethnic Groups, Minnesota Historical Society Press.

MOSTYN Trevor Éd. (1988) Cambridge Encyclopedia of the Middle East and North Africa, Cambridge Un. Press.

MOURA Paulo Cursino (1954) São Paulo de outrora: evocações da metrópole. S. Paulo, Liv. Martins Ed. SAFADY Jorge (1949) Coleção Brasil-Líbano-Síria, São Paulo, Editora Comercial Safady. 
SAFADY Jorge (s/d) O café e o mascate.

STEIN Stanley (1979) Origens e evolução da indústria têxtil no Brasil - 1850/1950. Rio de Janeiro, Campus.

TANNOUS Afif (1943) Acculturation of an Arab-Syrian Community in Deep South, American Sociological Review, v. 8 (3), p. 266.

TANNOUS Afif (1942) Emigration, A Force of Social Change in an Arab Village, Rural Sociology, vol. 7, pp. 62-74.

TOLEDO Benedito Lima (1987) Album Iconográfico da Avenida Paulista. S. Paulo, Ex Libris.

TRUZZI Oswaldo (1997b) The Right Place at the Right Time: Syrians and Lebanese in Brazil and the United States, a Comparative Approach. Journal of American Ethnic History, n 2, pp. 3-34.

TRUZZI Oswaldo (1992) De mascates a doutores : sírios e libaneses em São Paulo, São Paulo, Sumaré.

TRUZZI Oswaldo (1997a) Patrícios - Sírios e Libaneses em São Paulo, São Paulo, Hucitec.

WORCMAN Sara (1996) Do Tropical Inglês ao Blue Jeans (Projeto Memória do SAARA), Rio de Janeiro (exposition).

ZWEIG Stefan (1941) Brasil, País do Futuro. Rio de Janeiro, Ed. Guanabara.

\section{NOTES}

1. -La domination ottomane a duré dans cette région de 1516 à la fin de la Première Guerre mondiale.

2. -À la suite de la défaite des Turcs durant la Première Guerre mondiale, la France assuma le contrôle politique de cette région. Sous le régime de protectorat français, le Liban (capitale Beyrouth), une bande de terre étroite, au bord de la Méditerranée, à cette époque peuplée en grande majorité de chrétiens maronites, obtint son autonomie, par rapport au reste de la Syrie (capitale Damas) peuplée d'une majorité de musulmans. Les deux pays n'accédèrent à l'indépendance politique qu'en 1943 et 1946 (Mostyn 1988, Hitti 1951 et 1967).

3. -Cependant, il faut noter que l'immense majorité des émigrés était chrétienne, les musulmans préferaient émigrer vers certains pays africains (ou même du ProcheOrient) où ils pensaient pouvoir pratiquer leur religion plus librement que dans un pays catholique.

4. -Les flux retrouveront un niveau élevé après la Seconde Guerre mondiale.

5. -Le commerce ambulant a constitué aussi la première alternative d'insertion économique dans d'autres pays d'Amérique Latine (Bestene, 1992).

6. -Ce livre a été traduit en plus de trente langues. De plus d'une version cinématographique a été réalisée en 1983, le rôle de Nacib était tenu par l'acteur Marcello Mastroiani. 


\section{RÉSUMÉS}

Le but de cet article est de comprendre les principales caractéristiques des trajectoires des immigrants d'origine syrienne et libanaise au Brésil, entre la fin du XIX et les années cinquante. Plusieurs aspects sont abordés : causes du mouvement migratoire, intégration économique au Brésil comme colporteurs, affirmation du caractère commerçant de la colonie, transformations des images associées à ces immigrés, des identités et de la cohésion du groupe, et enfin mobilité sociale de la première génération née au Brésil. Les trajectoires des immigrants libanais et syriens dans la société brésilienne, et particulièrement à São Paulo, illustrent de façon exemplaire les opportunités offertes par une société en effervescence, devenant de plus en plus urbaine et complexe. Ces immigrants et leurs descendants ont bouleversé les structures sociales polarisées d'une société agraire en déclin, en inaugurant un nouveau ensemble de positions intermédiaires à l'intérieur du tissu social.

Lebanese and Syrian in Brazil, 1880-1950

This article focuses on explaining the main characteristics regarding the trajectories taken by Syrian and Lebanese immigrants to Brazil, between the last decades of the XIXth century and the fifties. The examined issues include the reasons for their coming, the beginning of their economic integration in Brazil as peddlers, their affirmation as merchants, the changes in their images, identities and group cohesion, and finally the patterns of upward mobility of the first Brazilian-born generation. Social trajectories of Syrian and Lebanese immigrants in Brazilian society (and particularly in São Paulo) stress how an increasingly urban and complex society could offer a set of opportunities for these newcomers. These immigrants and their descendants have challenged, in their way, the polarized structures of an decaying agrarian society, by founding and occupying a new set of intermediary positions in Paulista social structure.

Libaneses y sirios en Brasil, 1880-1950

Este articulo trata de entender las características mas importantes de las trayectorias de los inmigrantes de origen sirio o libanés en Brasil entre fines del siglo XIX y los años cincuenta. Los principales temas estudiados son las causas del movimiento migratorio, la integración económica inicial del grupo en la venta ambulante, la conquista de un nicho especializado en el comercio, las transformaciones de las representaciones asociadas a estos inmigrantes, de las identidades y de la cohesión del grupo, y por fin las principales vías de movilidad social recorridas por la primera generación nacida en Brasil. Las trayectorias de los inmigrantes sirios y libaneses en la sociedad brasileña, particularmente en Sao Paulo, ilustran de un modo ejemplar las oportunidades ofrecidas por una sociedad cada vez mas urbana, compleja y dinámica. A su manera, estos inmigrantes y sus descendientes trastornaron a las estructuras sociales polarizadas de una sociedad agraria en decadencia inaugurando, junto a otros grupos, un nuevo conjunto de posiciones intermediaria al interior del tejido social.

Libaneses e Sírios no Brasil entre 1880 e 1950

$\mathrm{O}$ artigo busca compreender as características mais importantes relativas às trajetórias de imigrantes de origem síria e libanesa no Brasil, entre o final do século XIX e os anos 50. As questões examinadas compreendem as causas do movimento migratório, a inserção econômica inicial do grupo como mascates, a conquista de um nicho de especialização como comerciantes, a transformação de suas identidades, imagens e diferenciação do grupo, e por fim as principais vias de mobilidade percorridas pela primeira geração nascida no Brasil. As trajetórias dos imigrantes sírios e libaneses na sociedade brasileira, e particularmente em São Paulo, ilustram de modo exemplar as oportunidades oferecidas por uma sociedade cada vez mais urbana, complexa e 
dinâmica. A seu modo, esses imigrantes e seus descendentes subverteram as estruturas sociais polarizadas de uma sociedade agrária em declínio, inaugurando, junto com outros grupos, um novo conjunto de posições intermediárias no interior do tecido social.

\section{AUTEUR}

\section{OSWALDO TRUZZI}

Docteur en Sciences Sociales, Professeur, l'Université Fédérale de São Carlos, Rod. Washington Luis, km. 235, 13595-905 São Carlos-SP-BRESIL. 